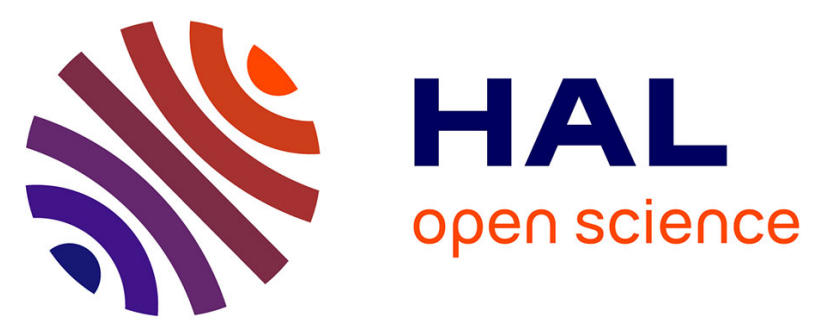

\title{
Expanded uncertainty associated with determination of isotope enrichment factors: Comparison of two point calculation and Rayleigh-plot
}

Maxime Julien, Alexis Gilbert, Keita Yamada, Richard J. Robins, Patrick Höhener, Naohiro Yoshida, Gérald S. Remaud

\section{To cite this version:}

Maxime Julien, Alexis Gilbert, Keita Yamada, Richard J. Robins, Patrick Höhener, et al.. Expanded uncertainty associated with determination of isotope enrichment factors: Comparison of two point calculation and Rayleigh-plot. Talanta, 2018, 176, pp.367-373. 10.1016/j.talanta.2017.08.038 . hal01581189

\section{HAL Id: hal-01581189 \\ https://hal-amu.archives-ouvertes.fr/hal-01581189}

Submitted on 3 May 2018

HAL is a multi-disciplinary open access archive for the deposit and dissemination of scientific research documents, whether they are published or not. The documents may come from teaching and research institutions in France or abroad, or from public or private research centers.
L'archive ouverte pluridisciplinaire HAL, est destinée au dépôt et à la diffusion de documents scientifiques de niveau recherche, publiés ou non, émanant des établissements d'enseignement et de recherche français ou étrangers, des laboratoires publics ou privés. 
1 Expanded uncertainty associated with determination of isotope enrichment

2 factors: comparison of two-points calculation and Rayleigh-plot

3 Maxime Julien ${ }^{1}$, Alexis Gilbert ${ }^{2,3}$, Keita Yamada ${ }^{1}$, Richard J. Robins ${ }^{4}$, Patrick

4 Höhener $^{5}$, Naohiro Yoshida ${ }^{1,3}$, Gérald S. Remaud ${ }^{4}$

$5{ }^{1}$ Department of Environmental Chemistry and Engineering, Tokyo Institute of 6 Technology, 4259 Nagatsuta-cho, Midori-ku, Yokohama, Kanagawa 226-8503, 7 Japan.

$8{ }^{2}$ Department of Earth and Planetary Sciences, Tokyo Institute of Technology, 9 Meguro, Tokyo 152-8551, Japan..

${ }^{3}$ Earth-Life Science Institute, Tokyo Institute of Technology, Meguro, Tokyo 1528551, Japan

${ }^{4}$ EBSI team, CEISAM, University of Nantes-CNRS UMR 6230, 2 rue de la Houssinière, F-44322 Nantes, France.

${ }^{5}$ Laboratoire Chimie Environnement, Aix Marseille University-CNRS UMR 7376, 3 place Victor Hugo, F-13331 Marseille, France.

Running title: Determination of enrichment factor and associated expanded uncertainty 
M Julien et al. - Talanta

\section{ABSTRACT}

The Enrichment Factor $(\varepsilon)$ is a common way to express Isotope Effects (IEs) associated with a phenomenon. Many studies determine $\varepsilon$ using a Rayleigh-plot, which needs multiple data points. More recent articles describe an alternative method using the Rayleigh equation that allows the determination of $\varepsilon$ using only one experimental point, but this method is often subject to controversy. However, a calculation method using two points (one experimental point and one at $t_{0}$ ) should lead to the same results because the calculation is derived from the Rayleigh equation. But, it is frequently asked "what is the valid domain of use of this two-points calculation?" The primary aim of the present work is a systematic comparison of results obtained with these two methodologies and the determination of the conditions required for the valid calculation of $\varepsilon$. In order to evaluate the efficiency of the two approaches, the expanded uncertainty $(U)$ associated with determining $\varepsilon$ has been calculated using experimental data from three published articles. The second objective of the present work is to describe how to determine the expanded uncertainty $(U)$ associated with determining $\varepsilon$. Comparative methodologies using both Rayleigh-plot and two-points calculation are detailed and it is clearly demonstrated that calculation of $\varepsilon$ using a single data point can give the same result as a Rayleighplot provided one strict condition is respected: that the experimental value is measured at a small fraction of unreacted substrate $(f<30 \%)$. This study will help stable isotope users to present their results in a more rigorous expression: $\varepsilon \pm U$ and therefore to define better the significance of an experimental results prior interpretation.

Capsule: Enrichment factor can be determined through two different methods and the calculation of associated expanded uncertainty allows checking its significance. 
M Julien et al. - Talanta

49

50 Keywords

51 Isotope Effects, isotope enrichment factor, expanded uncertainty, Rayleigh, two-

52 points

53 
M Julien et al. - Talanta

\section{INTRODUCTION}

Stable isotope analyses is widely used in different fields such as geochemistry [1], biology [2] or environmental sciences [3]. The isotope ratio of light elements $(\mathrm{C}, \mathrm{H}, \mathrm{O}$, $\mathrm{N}, \mathrm{S}$ or $\mathrm{Cl}$ ) is known to change through processes such as (bio)chemical reactions or physical processes due to preferential selection for the light or heavy isotope. This physical phenomenon, called the Isotope Effect (IE), can occur at equilibrium where heavy isotopes will be accumulated in one of the components of the system. Many processes such as liquid-vapor transformation can be associated with an Equilibrium Isotope Effect (EIE) [4]. IE can also be associated with a (bio)chemical reaction [5] where the presence of heavy isotopes can influence the reaction rate constant. Classically, this Kinetic Isotope Effect (KIE) is defined as the ratio of rate constants between two isotopes of a given element $\mathrm{KIE}=\left({ }^{\text {light }} \mathrm{k} /{ }^{\text {heavy }} \mathrm{k}\right)$ and depends on the mechanism of the reaction/process and on environmental parameters such as temperature or $\mathrm{pH}$ [6]. As a consequence, kinetic processes can be associated with an enrichment $(\mathrm{KIE}<1)$ or depletion $(\mathrm{KIE}>1)$ of heavy isotope of the reaction product(s) compared to the substrate(s). For a more detailed explanation, we recommended to read the guide "Practice and Principles of Isotopic Measurements in Organic Geochemistry" by J. M. Hayes where these concepts are explained in a very pedagogical way [7].

IE is not directly observable but it induces an alteration of isotopic abundance in the studied compounds: substrate(s) and/or product(s). This change in the isotope ratio, called isotopic fractionation, can be determined using isotope analysis and is often expressed as a fractionation factor $(\alpha)$ calculated from the equation described by Bigeleisen and Wolfsberg [8]. In the present article, isotopic fractionation has been expressed as an enrichment factor ( $\varepsilon$ [9] instead of $\alpha$ (where $\left.\varepsilon=(\alpha-1)^{\star} 1000\right)$. This 
notation has been chosen, because the sign of $\varepsilon$ directly informs on the IE associated with the studied process: when $\varepsilon$ is negative, the IE is considered as normal (light isotopes are preferentially used during the process) and when $\varepsilon$ is positive, the IE is inverse (transformation is faster using heavy isotopes). Enrichment factors can easily be determined using a Rayleigh-plot where $\varepsilon$ is the slope of the trend line $[10,11]$. Therefore, knowing the sign and amplitude of the IE allows conclusions to be drawn on the process and/or mechanism responsible for the production, degradation, or transformation of a given molecule. $\varepsilon$ (expressed in \%o) can be defined as follows:

$\varepsilon=\left({ }^{\text {heavy }} \mathrm{k} /{ }^{\text {light }} \mathrm{k}-1\right)^{*} 1000$

Note that in geo- and environmental sciences, in contrast to classical (bio)chemistry, the ratio of the heavy to light isotope (and not vice versa) is commonly used. However, what is usually measured during a given process is not directly the relative rate of the reaction but rather the isotope ratio of the starting material and the product at two or more points during the progress of the process/reaction. The link between isotope ratios, extent of progress of the reaction $f$ and $\varepsilon$ for a kinetic process is given by the Rayleigh equation [9, 12-14]:

$\mathrm{R} \simeq \mathrm{R}_{0} \times f^{\mathcal{E} / 1000}$

where, $\mathrm{R}_{0}$ is the isotope ratio at $\mathrm{t}_{0}, \mathrm{R}$ the isotope ratio at $\mathrm{t}_{f}$ and $f$ is the unreacted fraction of substrate at $t_{f}$. Equation 2 is a good approximation for all elements where the heavy isotope is much rarer than the light isotope. In cases of near equal abundance of isotopes, such as for B or when working with highly enriched compounds, equation 2 must be replaced by that proposed by Hunkeler et al. in 2002 [14].

The most common method for determining $\varepsilon$ is to use a Rayleigh-plot: the isotopic composition of the remaining reactant is measured at different fractions of unreacted 
substrate $(f)$ and $\ln \left(\mathrm{R} / \mathrm{R}_{0}\right)$ is plotted as a function of $\ln (f)[10,15]$. The enrichment factor $\varepsilon$ can then be obtained from the slope of a linear regression fitted to all data points. The variability of $\varepsilon$ is then expressed from the standard deviation of the slope. Most studies involving the determination of IEs have exploited this approach. In many situations, such as field sampling, measurement conditions do not allow isotope analysis at different times or different degrees of $f$ to be determined [16]. Nonetheless, an enrichment factor can still be obtained by using equation 2 above (Rayleigh equation). This calculation method only requires isotopic measurements on the starting substrate (at $\left.t=0, f_{0}=1\right)$ and on one data point $\left(\right.$ at $\left.t=t_{1}, f_{1}<1\right)$ : this calculation method is called two-points calculation in this article. The question is then: how to express the variability of the results?

The goal of the present article is to compare these two approaches, namely Rayleigh-plot and two-points calculation, both in terms of trueness and precision [17]. Note that these two $\varepsilon$ determination approaches are derived from the Rayleigh distillation equation, so they should lead to the same $\varepsilon$. We asked three fundamental questions: "what is the domain of use of the Rayleigh equation?", "how to express the uncertainty of the results from the two-points calculation mode?" and "what is the significance of the results based on the uncertainty determination in both $\varepsilon$ determination modes?". Three articles have been selected, with the authors' consent, to compare these two methods for the determination of the enrichment factor. Using data from these studies, we first compare $\varepsilon$ values obtained with these alternative approaches in order to evaluate under which conditions they give consistent results. In a second part, we detail how to determine the limit of significance of the calculated $\varepsilon$. Many studies only use the standard deviation (SD) of the isotopic measurement to indicate the significant threshold of the determination of $\varepsilon$. But this method does not 
129 include other sources of uncertainty such as the measurement of $f$, signal size or 130 linearity [18]. Calculation of the expanded uncertainty (named $U$ ) associated with the

131 determination of $\varepsilon$ is explained in the section below and a more detailed $U$ calculation

132 is described in the 'guide of expression of uncertainty in measurement' GUM [19].

133 Calculations of both $\varepsilon$ and its associated $U$ will help in determining IEs in a more 134 robust way and will provide a correct style to express the accuracy of the results.

\section{MATERIALS AND METHODS}

\section{Data collection}

138 Experimental data from three different articles have been collated, with the authors' consent, to compare $\varepsilon$ values obtained with Rayleigh-plot and the two-points 140 calculation and illustrate the advantage of performing the expanded uncertainty calculation. These are:

Article A: Gray J. R. et al., 2002, Environ. Sci. Technol., 36, 1931-1938 [20]

Article B: Cretnik S. et al., 2014, Molecules, 19, 6450-6473 [15]

Article C: Yamada K. et al., 2014, Rapid Commun. Mass Spectrom., 28, 1821-1828 [22]

These articles have been chosen in order to explore diverse isotope fractionation conditions (evaporation, biodegradation) and also to give examples with different

148 elements $\left({ }^{2} \mathrm{H},{ }^{13} \mathrm{C}\right.$, and $\left.{ }^{37} \mathrm{Cl}\right)$. Moreover, this selection includes an article using position-specific isotope analysis (PSIA) in order to confirm that these calculation methods are applicable in such a case. In the present work, the articles are called article $\mathrm{A}, \mathrm{B}$ or $\mathrm{C}$ to make the text easier to read. Note that in all three articles a Rayleigh-plot was used to calculate the isotope fractionation factor. 
153 A part of article A from Gray et al. 2002 [20] deals with the determination of both deuterium and carbon IEs associated with the aerobic biodegradation of methyl tertbutyl ether (MTBE) by bacteria strains discovered in a military base located in California. Bioremediation experiments have been performed in vitro using sediments and groundwater extracted from the contaminated site. Stable isotope analysis was performed by Gas Chromatography - Combustion - isotope ratio monitoring by Mass Spectrometry (irm-GC/MS) [21] and the amount of unreacted substrate was measured by gas chromatography for each experimental point.

In article B, Cretnik et al. 2014 [15] report a study of the in vitro anaerobic biodegradation of tetrachloroethene (PCE) by Desulfitobacterium sp. strain Viet1. Both carbon and chlorine isotope ratios were monitored throughout the biotransformation using irm-GC/MS and $f$ was determined by gas chromatography.

Article C, Yamada et al. 2014 [22], deals with position-specific isotope analysis of acetic acid using on-line pyrolysis combined with GC-C-irm-MS (Py-irm-GC/MS) [23]. The authors determined position-specific isotope effects (PSIEs) associated with the evaporation of acetic acid. For this experiment, 14 vials containing pure acetic acid were placed in a hermetically-sealed chamber containing sodium hydroxide $(\mathrm{NaOH})$ to remove evaporated acetic acid. Vials are weighted at $t_{0}$ and at $t_{f}$ in order to determine $f$ and PSIA were performed on each sample at variable $f$.

\section{Determination of the enrichment factor $(\varepsilon)$}

The two methods for $\varepsilon$ determination are described in this section.

The most common method is the determination of $\varepsilon$ using a Rayleigh-plot, in which $\ln (f)$ is plotted on the $x$-axis and $\ln \left(R / R_{0}\right)$ on the $y$-axis $[10,11]$. In this graph, the enrichment factor $(\varepsilon)$ corresponds to the slope of the trend line and the associated 
177 root mean square $\left(\mathrm{R}^{2}\right)$ gives a first indication of the quality of the linearity. Note that 178 the regression is not forced through the origin following a recommendation by Scott 179 et al. in 2004 [24].

180 The second method is the determination of $\varepsilon$ with the two-points calculation and has 181 been performed using the following relationship [25], in which the isotope ratios $\mathrm{R}$ (as 182 in equation 2) are expressed as isotopic composition $\delta$ :

$\varepsilon=\ln \left(\frac{\delta^{\mathrm{A}} \mathrm{X}_{\mathrm{t} f}+1000}{\delta^{\mathrm{A}} \mathrm{X}_{\mathrm{t} 0}+1000}\right)\left(\frac{1000}{\ln f}\right)$

$187 \varepsilon=\frac{\Delta \delta^{\mathrm{A} X}}{\ln f}$

where $\delta^{A} X_{t 0}$ and $\delta^{A} X_{t f}$ are respectively the isotopic composition of the starting reactant (initial state) and the remaining reactant at $t_{f}$. This relationship can be approximated as described in the following equation [4]:

where $\Delta \delta^{A} X$ stands for the isotopic fractionation between the reactant at $t_{0}$ and at time $f\left(\Delta \delta^{A} X=\delta^{A} X_{t f}-\delta^{A} X_{t 0}\right)$. These two equations give very similar results [9] and the simplified equation 4 is employed here for the determination of $\varepsilon$ with results from the selected articles.

\section{Determination of expanded uncertainty (U)}

In order to determine the significance threshold of the enrichment factor, the expanded uncertainty needs to be calculated. This article is the first which offers a fully-described expanded uncertainty calculation method within this context.

The determination of the expanded uncertainty associated with the enrichment factor determined using the Rayleigh-plot can be directly calculated using the function "LINEST" in Microsoft Excel ${ }^{\mathrm{TM}}$, as described by Elsner et al. [10]. This function 
199

200

201

202

203

calculates both the slope and the standard deviation of the trend line (STDV slope).

The expanded uncertainty can thus be calculated as follows:

$\mathrm{U}=\mathrm{k} \times \mathrm{STDV}$ slope

where $\mathrm{k}=2$ for a coverage factor of $95 \%$ (t value in Student table).

In the case where $\varepsilon$ is determined with a single data point, $U$ can be calculated from the uncertainty estimation described in the 'guide of expression of uncertainty in measurement' GUM [19]. This evaluation criterion has been chosen because all parameters that can affect the final result are taken into account by $U$ calculation, unlike in most articles in which authors only use the SD of the isotope analysis method as described by the instrument manufacturer. Parameters that need to be considered in the calculation of expanded uncertainty can be assembled in an Ishikawa diagram (also called fishbone diagram or cause-and-effect diagram, see Figure 1). Two contributions have to be taken into account, (i) the isotopic measurement of both the starting reactant $\left(a t t_{0}\right)$ and the remaining reactant $\left(a t t_{f}\right)$ along with the standard deviation of the measurements and (ii) the determination of $f$ (and its associated variability) which can be performed by weighting, chromatography or spectroscopy (like NMR or Mass Spectrometry).

The expanded uncertainty can be calculated using the following equation:

$$
\mathrm{U}=\mathrm{k} \times \mathcal{E} \times \sqrt{\left(\frac{\mathrm{u}\left(\delta^{\mathrm{A} X}\right)}{\Delta \delta^{\mathrm{A} X}}\right)^{2}+\left(\frac{\mathrm{u}(\mathrm{p})}{\mathrm{p}}\right)^{2}}
$$

where $\mathrm{k}=2$ for a coverage factor of $95 \%$ and $\mathrm{p}$ is the quantity measured during $f$ determination (a mass, an area). Then, $u\left(\delta^{A} X\right)$ is the uncertainty associated with isotopic measurement at $t_{0}$ and $t_{f}$ obtained by the propagation uncertainty law:

$$
\mathrm{u}\left(\delta^{\mathrm{A}} \mathrm{X}\right)=\sqrt{\mathrm{SD}^{2}+\mathrm{SD}^{2}}=\sqrt{2 \times \mathrm{SD}^{2}}
$$


222 where SD is the standard deviation of the isotopic measurement. The analyte at the

223 initial and the final states is measured using strictly the same protocol, i.e. there is no

224 linearity problem. The next step is the calculation of $u(p)$, namely the precision of the

225 analytical method used to determine $f$, which is expressed as an uncertainty using a 226 rectangular distribution (also called continuous uniform distribution) [19] and 227 calculated using the following equation:

$228 \mathrm{u}(\mathrm{p})=\sqrt{\left(\frac{\mathrm{v}}{\sqrt{3}}\right)^{2} \times \mathrm{k}}$

where $\mathrm{v}$ is the standard deviation of the analytical method used to determine $f$

230 (balance, spectrometric technique), $\mathrm{p}$ is the quantity measured (mass, area) and $\mathrm{k}=$ 2312 for a coverage factor of $95 \%$.

232 In the general equation for $U$ calculation (equation 6 ), the term $\left(\frac{u(p)}{p}\right)^{2}$ is generally negligible but needs to be taken into account in order to ensure that every step of the uncertainty calculation is performed in a correct way. The precision of $f$ determination

235 needs to be taken into account during $\varepsilon$ determination, because both the measurement method and the sampling can be associated with a systematic error [26].

Once the enrichment factor and its associated expanded uncertainty are calculated,

239 the final result is expressed as $\varepsilon \pm U$. This expanded uncertainty value is slightly overestimated but covers all sources of variability. In these conditions, the observed 241 isotope effect is considered significant when $|\varepsilon|>|U|$. 
245 In this study, $\varepsilon$ and the associated expanded uncertainty are calculated for each data

point of the selected articles and the values are compared with those obtained using Rayleigh-plots. In this section, the studied element is specified in enrichment factor expression in order to make the reading easier (i.e. $\varepsilon_{\mathrm{C}}$ in the case of ${ }^{13} \mathrm{C}$ enrichment factor).

Figure 2 shows the Rayleigh-plots obtained for the datasets used in this study. In most cases, the Rayleigh-plot trend-line fits very well with the experimental points, with root mean square values $\left(R^{2}\right)$ higher than 0.98 , which clearly demonstrates the linearity of the experimental data (Figure 2). The only exception is the trend line obtained from the monitoring of $\delta^{13} \mathrm{C}$ of the carboxyl group of acetic acid during evaporation (Figure 2c) for which $\mathrm{R}^{2}$ is about zero. This value is not surprising given that the slope of the curve is 0 (implying that there is no significant isotope fractionation associated with evaporation on this carbon-atom position).

For each dataset, $\varepsilon$ was determined using the slope of the trend line. Values for $\varepsilon$ obtained from each data point using the two-points calculation (equation 3) are presented in Table 1. In most cases, two-points calculation method gives very similar results to those from Rayleigh-plots, but only for small values of $f$ (which corresponds to the fraction of unreacted substrate at $t_{f}$ ). As an example, results from article $A$ (Figure 2a) show a very high $\varepsilon_{H}$ of $-65.7 \%$ obtained from Rayleigh-plot and twopoints calculations give values comprised between -63.0 and $-69.3 \%$ when $f$ is lower than $20 \%$. The same phenomenon is observed in the case of ${ }^{13} \mathrm{C}$ with an $\varepsilon_{\mathrm{c}}$ of $-1.5 \%$ according to Rayleigh-plot and very different values obtained with equation 3 when $f$ $>24 \%$. Rayleigh-plots drawn using experimental data from article B (Figure 2b) show an $\varepsilon_{\mathrm{Cl}}$ value of $-5.0 \%$ and an $\varepsilon_{\mathrm{c}}$ of $-19.3 \%$. These data also demonstrate the trueness of $\varepsilon$ calculated using equation 3 , with very similar values obtained when $f<34.8 \%$ in 
270 the case of ${ }^{37} \mathrm{Cl}$ and $f<31.1 \%$ for ${ }^{13} \mathrm{C}$. Figure $2 \mathrm{c}$ demonstrates that the two-points

271 calculation method is also efficient with PSIA data. Rayleigh-plot results from article

$272 \mathrm{C}$ indicate that acetic acid evaporation is associated with a $\boldsymbol{\varepsilon}_{\mathrm{C} \text {-Bulk }}$ of $-1.0 \%$ mostly

273 located on the methyl group $\left(\varepsilon_{c-M e t h y l}=-2.0 \%\right.$ ) which is confirmed by the comparable

274 values obtained using the two-points calculation when $f<39.0 \%$. Calculated $\varepsilon$ values

275 also confirm the absence of significant isotope effect located on the carboxyl

276 function.

277 According to data from Figure 2 and Table 1, the two methods seem to be exploitable

278 to determine $\varepsilon$ from varied experimental data and elements. The limit of the two279 points calculation is the value of $f$, but this limit is not clearly established. 280 Determination of expanded uncertainty should help to fix the limit of the domain of 281 use of the Rayleigh equation.

\section{Expanded uncertainty}

283 Expanded uncertainties associated with $\varepsilon$ determination using both Rayleigh-plot and two-points calculation are detailed in Table 1 . When $\varepsilon$ is determined through Rayleigh-plot using experimental data from Article $A$, the expanded uncertainty associated with ${ }^{13} \mathrm{C}$ IE determination is very small $(U=0.1 \%$ ) compared to $\varepsilon$. That result suggests that $\varepsilon$ is significant. The $U$ value is higher in the case of ${ }^{2} \mathrm{H}(3.7 \%$ ) but this result is in accordance with the higher $\varepsilon_{H}$ of $-65.7 \%$ detected through deuterium isotope analysis. These two examples demonstrate that an IE can be considered as significant even if its associated uncertainty is high; when $|\varepsilon|>|U|$ (or $|U / \varepsilon|<1$ ),

291 the result is significant. Carbon-13 bulk enrichment factors obtained in the other articles are also significant with $\varepsilon_{C}=-19.3 \pm 0.8 \%$ in article $B, \varepsilon_{C-B u l k}=-1.0 \pm 0.1 \%$ o and $\varepsilon_{\mathrm{C}-\text { Methyl }}=-2.0 \pm 0.1 \%$ in article $\mathrm{C}$ (Table 1$)$. The same conclusion can be made with results from ${ }^{37} \mathrm{Cl}$ experimental data of article $\mathrm{B}$ with $\varepsilon_{\mathrm{Cl}}=-5.0 \pm 0.2 \%$. 
M Julien et al. - Talanta

295 Enrichment factors calculated using the two-points approach are similar to those from 296 Rayleigh-plots, as previously described by Jeannottat and Hunkeler [25], but $U$

297

298 obtained with the two methods are very different and $U$ seems to be increased with $f$ (Table 1), which is coherent with the observations made above. In each study, when the process is not well advanced ( $f>30 \%$ ), the equation gives different $\varepsilon$ results than those obtained with the Rayleigh-plot. When less than $70 \%$ of substrate has reacted, the calculated $\varepsilon$ is not significant, as demonstrated in Figure $3 \mathrm{~b}$ with $\left|\mathrm{U} / \varepsilon_{\text {Rayleigh }}\right|>1$. For instance, $\varepsilon_{\mathrm{C}-\text { Bulk }}$ is $-1.0 \pm 0.1 \%$ (article C) according to the Rayleigh-plot and the same value is obtained from the two-points calculation with $\left|U / \varepsilon_{\text {Rayleigh }}\right|$ comprised between 0.3 and $0.7 \%$ provided $f<30 \%$ (Figure $3 \mathrm{~b}$ ).

From all the data studied here, data points calculated at $f>30 \%$ give different values from those determined using Rayleigh-plot and this difference is confirmed by high expanded uncertainty values. The determination of $|\mathrm{U} / \varepsilon|$ is an excellent tool to ensure that the uncertainties associated with the determination of IE do not exceed the measured $\varepsilon$ [27].

\section{Significance of the results}

The results obtained from each article were used to draw three graphs (Figure 3) in order to evaluate the trueness of the $\varepsilon$ determined with the two-points calculation and the minimum of substrate transformation that must be used to ensure that results are significant.

The variation of the expanded uncertainty depending on the amount of remaining substrate is described in Figure 3a. This result confirms the need to set a limit of $f$ value in order to use the two-points calculation in a correct way. The graph presented in Figure $3 b$ shows the correlation between the reaction yield $(1-f)$ and the degree of significance of $\varepsilon$ calculated using the two-points method (the result is considered 
M Julien et al. - Talanta

320

321

322

323

324

325

326

327

328

329

330

331

332

333

334

335

336

337

338

as significant when $|U / \varepsilon|<1$ ). These data mean that results obtained with the twopoints calculation are significant on a large range of transformation yields, even if the expanded uncertainty associated with the enrichment factor increases with the amount of remaining substrate. This graph demonstrates that the results obtained are significant when $1-f>0.7$ ( $f<30 \%)$. The relationship between the transformation yield $(1-f)$ and the gap between $\varepsilon$ values obtained with the two methods $\left(\left(\varepsilon-\varepsilon_{\text {Rayleigh }}\right) / \varepsilon_{\text {Rayleigh }}\right)$ clearly demonstrates the trueness of $\varepsilon$ values determined with the two-points calculation (see Figure 3c), considering that the Rayleigh-plot allows the calculation of a true $\varepsilon$. Indeed, values obtained using the two-points method have a deviation of $10 \%$ relative to the true value ( $\left.\varepsilon_{\text {Rayleigh }}\right)$ when $f$ $<20 \%(1-f>0.8)$ and a deviation of $20 \%$ when $f<30 \%(1-f>0.7)$, which means that the two-points method allows the calculation of true values of $\varepsilon$ when the selected data point has been measured at high transformation yield.

These results demonstrate that the two-points calculation method is efficient when the amount of remaining substrate is below $30 \%$ (which corresponds to a reaction yield of $70 \%)$.

\section{CONCLUSIONS}

Considering these observations, the determination of $\varepsilon$ using a single experimental point can be justified in conditions wherein the yield of the studied transformation is above $70 \%(f<30 \%)$. Within this range, the $\varepsilon$ values calculated are similar to those obtained from Rayleigh-plots, and so is the associated uncertainty. Furthermore, expanded uncertainty calculations are shown as an excellent and rigorous way to check if the determined values of $\varepsilon$ are significant and, in particular, are a good tool to assess the validity of the two-points calculation. 
345 Enrichment factors can therefore be directly determined from experimental data using two different methods. The classical Rayleigh-plot approach is very efficient and the resulting $\varepsilon$ associated expanded uncertainty can be calculated using the function "LINEST" of Microsoft Excel ${ }^{\mathrm{TM}}$. Equations that allow calculating enrichment factor with only one experimental point can be very useful when it is difficult to collect several samples during a transformation. When using this method, it is recommended to work with transformations of more than $70 \%$.

For both methods, determining the expanded uncertainty associated with a result is highly recommended in order to draw any conclusion about the studied phenomenon. This calculation requires the determination of precision for both the isotopic measurement and the yield. In these conditions, the final result will take into account all uncertainty sources associated with both the experiments and the measurements. The final IE result can be expressed as $\varepsilon \pm U$ and considered as significant when $|\varepsilon|>|U|$ (or $|U / \varepsilon|<1)$. In these conditions, the two-points calculation is an excellent way to determine the IE associated with a transformation and the use of the expanded uncertainty reinforces the validity of conclusions that can be made about the studied mechanism.

Such calculations can be complex to set up; so this article provides a spreadsheet in which both $\varepsilon$ and $U$ can be automatically calculated after appropriate input: the initial isotopic composition $(\mathrm{t}=0)$, the isotopic composition at $\mathrm{t}=f$, the isotopic measurement precision (SD), the transformation yield $(1-f)$, the quantification measurement (mass or area) and the precision associated with $f$ determination.

\section{Supporting information available}


M Julien et al. - Talanta

EXCEL spreadsheet for calculation of enrichment factor and uncertainty by the twopoint method.

\section{Acknowledgment}

M. Julien thanks the "Région Pays de la Loire" for funding his postdoctoral bursary through the project PLAISIR. A. Gilbert thanks the Grant-in-Aid for Young Scientist (B) (15K17774) and the Grant-in-Aid for Scientific Research (S) (23224013), MEXT, Japan, for financial support. The authors thank Prof. Barbara Sherwood-Lollar and Dr. Martin Elsner for agreeing to share their experimental data.

\section{References}

[1] E.M. Galimov, Isotope organic geochemistry, Org. Geochem. 37 (2006) 12001262.

[2] J.M. Hayes, Fractionation of Carbon and Hydrogen Isotopes in Biosynthetic Processes, Rev. Min. Geochem. 43 (2001) 225-277.

[3] M. Elsner, M.A. Jochmann, T.B. Hofstetter, D. Hunkeler, A. Bernstein, T.C. Schmidt, A. Schimmelmann, Current challenges in compound-specific stable isotope analysis of environmental organic contaminants, Anal. Bioanal. Chem. 403 (2012) 2471-91.

[4] M. Julien, J. Parinet, P. Nun, K. Bayle, P. Höhener, R.J. Robins, G.S. Remaud, Fractionation in position-specific isotope composition during vaporization of environmental pollutants measured with isotope ratio monitoring by ${ }^{13} \mathrm{C}$ nuclear magnetic resonance spectrometry, Environ. Pollut. 205 (2015) 299-306.

[5] M.J. DeNiro, S. Epstein, Mechanism of carbon isotope fractionation associated with lipid synthesis, Science 197 (1977) 261-263.

[6] M. Elsner, L. Zwank, D. Hunkeler, R.P. Schwarzenbach, A New Concept Linking Observable Stable Isotope Fractionation to Transformation Pathways of Organic Pollutants, Environ. Sci. Technol. 39 (2005) 6896-6916.

[7] J.M. Hayes, Practice and principles of isotopic measurements in organic geochemistry, in: A. Sessions 2002,<http://web.gps.caltech.edu/ als/research articles/other stuff/hayespnp.pdf>. [8] J. Bigeleisen, M. Wolfsberg, Theoretical and Experimental Aspects of Isotope Effects in Chemical Kinetics, Advances in Chemical Physics, John Wiley \& Sons, Inc.2007, pp. 15-76.

[9] A. Mariotti, J.C. Germon, P. Hubert, P. Kaiser, R. Letolle, A. Tardieux, P. Tardieux, Experimental determination of nitrogen kinetic isotope fractionation: Some principles; illustration for the denitrification and nitrification processes, Plant and Soil 62 (1981) 413-430. 
[10] M. Elsner, J. McKelvie, G. Lacrampe Couloume, B. Sherwood Lollar, Insight into Methyl tert-Butyl Ether (MTBE) Stable Isotope Fractionation from Abiotic Reference Experiments, Environ. Sci. Technol. 41 (2007) 5693-5700.

[11] P. Höhener, O. Atteia, Rayleigh equation for evolution of stable isotope ratios in contaminant decay chains, Geochim. Cosmochim. Acta 126 (2014) 70-77.

[12] C. Kendall, J.J. Mc Donnell, Isotope tracers in cachment hydrology, Elsevier Science B.V., Amsterdam1998.

[13] M.C. Aelion, P. Höhener, D. Hunkeler, R. Aravena, Environmental Isotopes in Biodegradation and Bioremediation, Taylor \& Francis Group2010.

[14] D. Hunkeler, Quantification of Isotope Fractionation in Experiments with Deuterium-Labeled Substrate, Appl. Environ. Microbiol. 68 (2002) 5205-5207.

[15] S. Cretnik, A. Bernstein, O. Shouakar-Stash, F. Löffler, M. Elsner, Chlorine Isotope Effects from Isotope Ratio Mass Spectrometry Suggest Intramolecular C-Cl Bond Competition in Trichloroethene (TCE) Reductive Dehalogenation, Molecules 19 (2014) 6450.

[16] D.B. McNevin, M.R. Badger, H.J. Kane, G.D. Farquhar, Measurement of (carbon) kinetic isotope effect by Rayleigh fractionation using membrane inlet mass spectrometry for $\mathrm{CO}_{2}$-consuming reactions, Functional Plant Biology 33 (2006) 11151128.

[17] T.B. Coplen, Guidelines and recommended terms for expression of stableisotope-ratio and gas-ratio measurement results, Rapid Commun. Mass Spectrom. 25 (2011) 2538-2560.

[18] B. Sherwood Lollar, S.K. Hirschorn, M.M.G. Chartrand, G. Lacrampe-Couloume, An Approach for Assessing Total Instrumental Uncertainty in Compound-Specific Carbon Isotope Analysis: Implications for Environmental Remediation Studies, Anal. Chem. 79 (2007) 3469-3475.

[19] JCGM/WG, Evaluation of measurement data- Guide to the expression of uncertainty in measurement. Evaluation of measurement data- Guide to the expression of uncertainty in measurement 100., 2008.

[20] J.R. Gray, G. Lacrampe-Couloume, D. Gandhi, K.M. Scow, R.D. Wilson, D.M. Mackay, B. Sherwood Lollar, Carbon and Hydrogen Isotopic Fractionation during Biodegradation of Methyl tert-Butyl Ether, Environ. Sci. Technol. 36 (2002) 19311938.

[21] J.T. Brenna, T.N. Corso, H.J. Tobias, R.J. Caimi, High-precision continuous-flow isotope ratio mass spectrometry, Mass Spectrom. Rev. 16 (1997) 227-258.

[22] K. Yamada, M. Kikuchi, A. Gilbert, N. Yoshida, N. Wasano, R. Hattori, S. Hirano, Evaluation of commercially available reagents as a reference material for intramolecular carbon isotopic measurements of acetic acid, Rapid Commun. Mass Spectrom. 28 (2014) 1821-1828.

[23] T.N. Corso, J.T. Brenna, High-precision position-specific isotope analysis, Proc. Natl. Acad. Sci. 94 (1997) 1049-1053.

[24] K.M. Scott, X. Lu, C.M. Cavanaugh, J.S. Liu, Optimal methods for estimating kinetic isotope effects from different forms of the Rayleigh distillation equation, Geochim. Cosmochim. Acta 68 (2004) 433-442.

[25] S. Jeannottat, D. Hunkeler, Chlorine and Carbon Isotopes Fractionation during Volatilization and Diffusive Transport of Trichloroethene in the Unsaturated Zone, Environ. Sci. Technol. 46 (2012) 3169-3176.

[26] D. Buchner, B. Jin, K. Ebert, M. Rolle, M. Elsner, S.B. Haderlein, Experimental Determination of Isotope Enrichment Factors - Bias from Mass Removal by Repetitive Sampling, Environ. Sci. Technol. 51 (2017) 1527-1536. 
457

458

459

460

461

[27] D.A. Singleton, A.A. Thomas, High-Precision Simultaneous Determination of Multiple Small Kinetic Isotope Effects at Natural Abundance, J. Am. Chem. Soc. 117 (1995) 9357-9358.

\section{Graphical abstract}

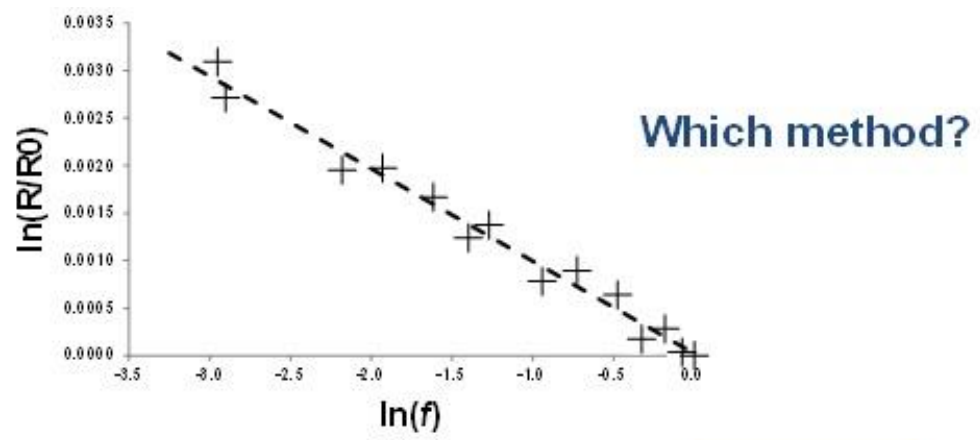

$$
\varepsilon=\frac{\Delta \delta^{13} \mathrm{C}}{\ln (f)}
$$

How to calculate?

\section{$\varepsilon \pm U$}

Is it significant?

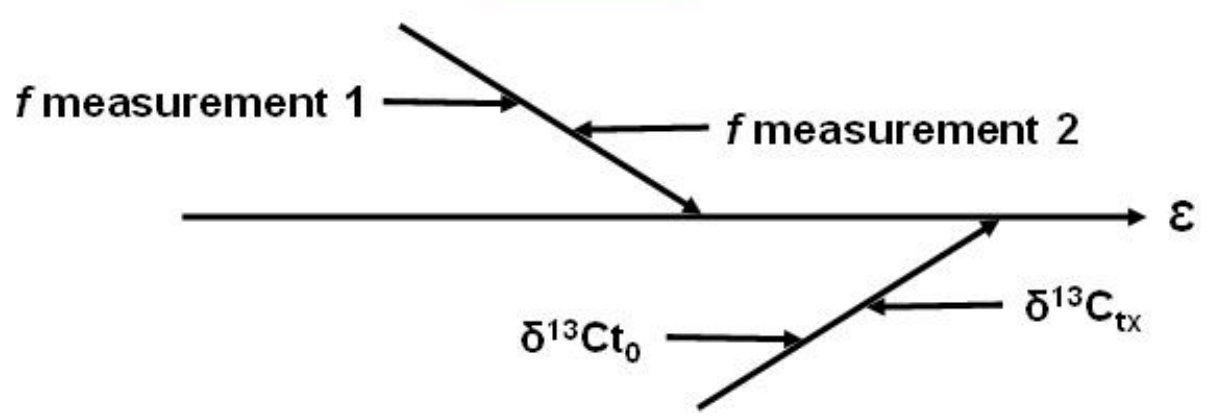


475 Table 1: Values of $\varepsilon$ calculated using each experimental point from (a) Gray et al. 476 2002, (b) Cretnik et al. 2014 and (c) Yamada et al. 2014. Associated expanded 477 uncertainties $(U)$ are also listed and compared to $\varepsilon$ values obtained from Rayleigh478 plots.

\begin{tabular}{|c|c|c|c|}
\hline \multirow[b]{2}{*}{ a) } & \multicolumn{3}{|c|}{${ }^{13} \mathrm{C}$} \\
\hline & $f$ & $\varepsilon(\% 0)$ & U (\%o) \\
\hline & $100.0 \%$ & - & - \\
\hline & $80.0 \%$ & 0.0 & 6.3 \\
\hline & $48.7 \%$ & -1.0 & 2.0 \\
\hline & $42.6 \%$ & -0.9 & 1.7 \\
\hline & $33.9 \%$ & -1.2 & 1.3 \\
\hline & $31.3 \%$ & -1.1 & 1.2 \\
\hline & $26.1 \%$ & -1.2 & 1.1 \\
\hline & $23.5 \%$ & -1.4 & 1.0 \\
\hline & $19.1 \%$ & -1.3 & 0.9 \\
\hline & $15.7 \%$ & -1.4 & 0.8 \\
\hline & $8.7 \%$ & -1.4 & 0.6 \\
\hline & $8.7 \%$ & -1.6 & 0.6 \\
\hline & $5.2 \%$ & -1.4 & 0.5 \\
\hline & $4.3 \%$ & -1.5 & 0.5 \\
\hline & $3.5 \%$ & -1.4 & 0.4 \\
\hline & $2.6 \%$ & -1.4 & 0.4 \\
\hline & $0.9 \%$ & -1.4 & 0.3 \\
\hline & $0.3 \%$ & -1.4 & 0.4 \\
\hline & $\begin{array}{l}\text { Rayleigh } \\
\text { plot }\end{array}$ & -1.5 & 0.1 \\
\hline
\end{tabular}

\begin{tabular}{ccc}
\multicolumn{3}{c}{${ }^{2} \mathrm{H}$} \\
\hline$f$ & $\varepsilon(\%$ d & $U(\%)$ \\
\hline $100.0 \%$ & - & - \\
$97.7 \%$ & -42.8 & 484.2 \\
$80.4 \%$ & -45.9 & 51.9 \\
$64.3 \%$ & -29.4 & 25.6 \\
$19.3 \%$ & -68.7 & 6.9 \\
$18.8 \%$ & -62.9 & 6.8 \\
$12.9 \%$ & -69.3 & 5.5 \\
$9.2 \%$ & -64.1 & 4.8 \\
$6.4 \%$ & -67.4 & 4.2 \\
$3.2 \%$ & -67.5 & 3.8 \\
$1.6 \%$ & -63.0 & 4.4 \\
\hline Rayleigh & -65.7 & 3.7 \\
plot & &
\end{tabular}

b)

\begin{tabular}{ccc}
\multicolumn{3}{c}{${ }^{13} \mathrm{C}$} \\
\hline$f$ & $\varepsilon(\%)$ & $U(\%)$ \\
\hline $100.0 \%$ & - & - \\
$92.3 \%$ & -15.5 & 17.5 \\
$83.7 \%$ & -13.4 & 8.0 \\
$76.6 \%$ & -6.8 & 5.3 \\
$71.7 \%$ & -13.1 & 4.2 \\
$70.4 \%$ & -11.7 & 4.0 \\
$68.5 \%$ & -14.4 & 3.7 \\
$61.0 \%$ & -17.7 & 2.9 \\
$56.1 \%$ & -14.0 & 2.4 \\
$49.0 \%$ & -15.6 & 2.0 \\
$34.8 \%$ & -16.7 & 1.3 \\
$31.1 \%$ & -16.9 & 1.2 \\
$14.6 \%$ & -18.6 & 0.8 \\
$7.8 \%$ & -18.9 & 0.7 \\
\hline Rayleigh & -19.3 & 0.8 \\
plot & &
\end{tabular}

c)

\begin{tabular}{ccccc}
\hline$f$ & $\varepsilon_{\text {bulk }}(\%)$ & $\varepsilon_{\text {methyl }}(\%$ ) & $\varepsilon_{\text {carboxyl }}(\% \circ)$ & $U(\% \circ)$ \\
\hline $100.0 \%$ & - & - & - & - \\
$92.7 \%$ & -0.4 & -0.6 & -0.3 & 11.2 \\
$83.4 \%$ & -1.5 & -3.4 & 0.4 & 4.7 \\
$72.2 \%$ & -0.5 & -1.3 & 0.3 & 2.6 \\
$62.2 \%$ & -1.3 & -1.7 & -0.9 & 1.8 \\
$48.3 \%$ & -1.2 & -1.3 & -1.1 & 1.2 \\
$39.0 \%$ & -0.8 & -1.8 & 0.2 & 0.9 \\
$28.0 \%$ & -1.0 & -1.7 & -0.4 & 0.7 \\
$24.7 \%$ & -0.9 & -2.1 & 0.4 & 0.6 \\
$19.9 \%$ & -1.0 & -1.8 & -0.2 & 0.5 \\
$14.5 \%$ & -1.0 & -1.9 & -0.1 & 0.4 \\
$11.3 \%$ & -0.9 & -1.9 & 0.2 & 0.4 \\
$5.5 \%$ & -0.9 & -1.9 & 0.1 & 0.3 \\
$5.2 \%$ & -1.0 & -1.8 & -0.2 & 0.3 \\
\hline & -1.0 & - & - & 0.1 \\
slope & - & -2.0 & - & 0.2 \\
& - & - & 0.0 & 0.1
\end{tabular}

\begin{tabular}{ccc}
\multicolumn{3}{c}{${ }^{37} \mathrm{Cl}$} \\
\hline $\boldsymbol{f}$ & $\mathcal{\varepsilon}(\% \circ)$ & $\mathrm{U}(\% \circ)$ \\
\hline $100.0 \%$ & - & - \\
$92.3 \%$ & -5.7 & 7.0 \\
$83.7 \%$ & -6.0 & 3.2 \\
$76.6 \%$ & -2.3 & 2.1 \\
$71.7 \%$ & -4.2 & 1.7 \\
$70.4 \%$ & -3.8 & 1.6 \\
$68.5 \%$ & -4.6 & 1.5 \\
$61.0 \%$ & -4.8 & 1.1 \\
$56.1 \%$ & -4.1 & 1.0 \\
$49.0 \%$ & -4.5 & 0.8 \\
$34.8 \%$ & -4.6 & 0.5 \\
$31.1 \%$ & -4.8 & 0.5 \\
$14.6 \%$ & -5.3 & 0.3 \\
$7.8 \%$ & -4.9 & 0.2 \\
\hline Rayleigh & -5.0 & 0.2 \\
plot & &
\end{tabular}


Figure 1: Ishikawa diagram describing all sources of uncertainty in the case of isotopic measurements of the studied compound at $t_{0}$ and $t_{x}$ and two measurements required to determine $f$.

492

493

494 495

496

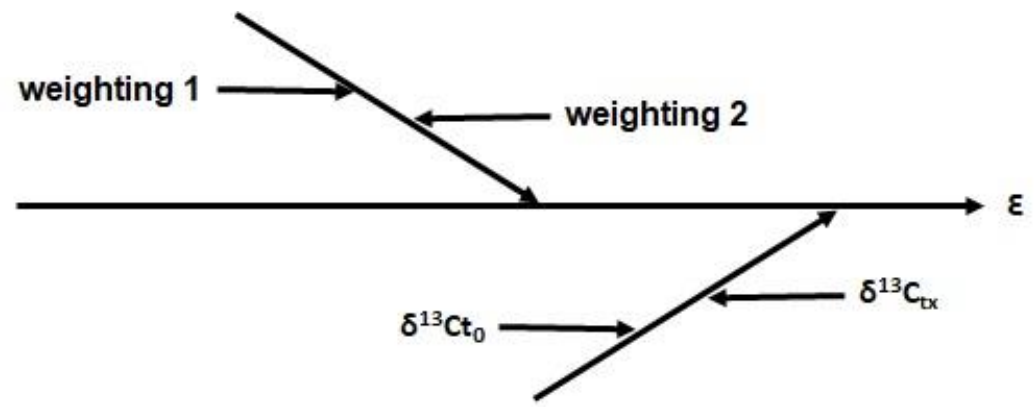
Cretnik et al. 2014 and (c) Yamada et al. 2014.
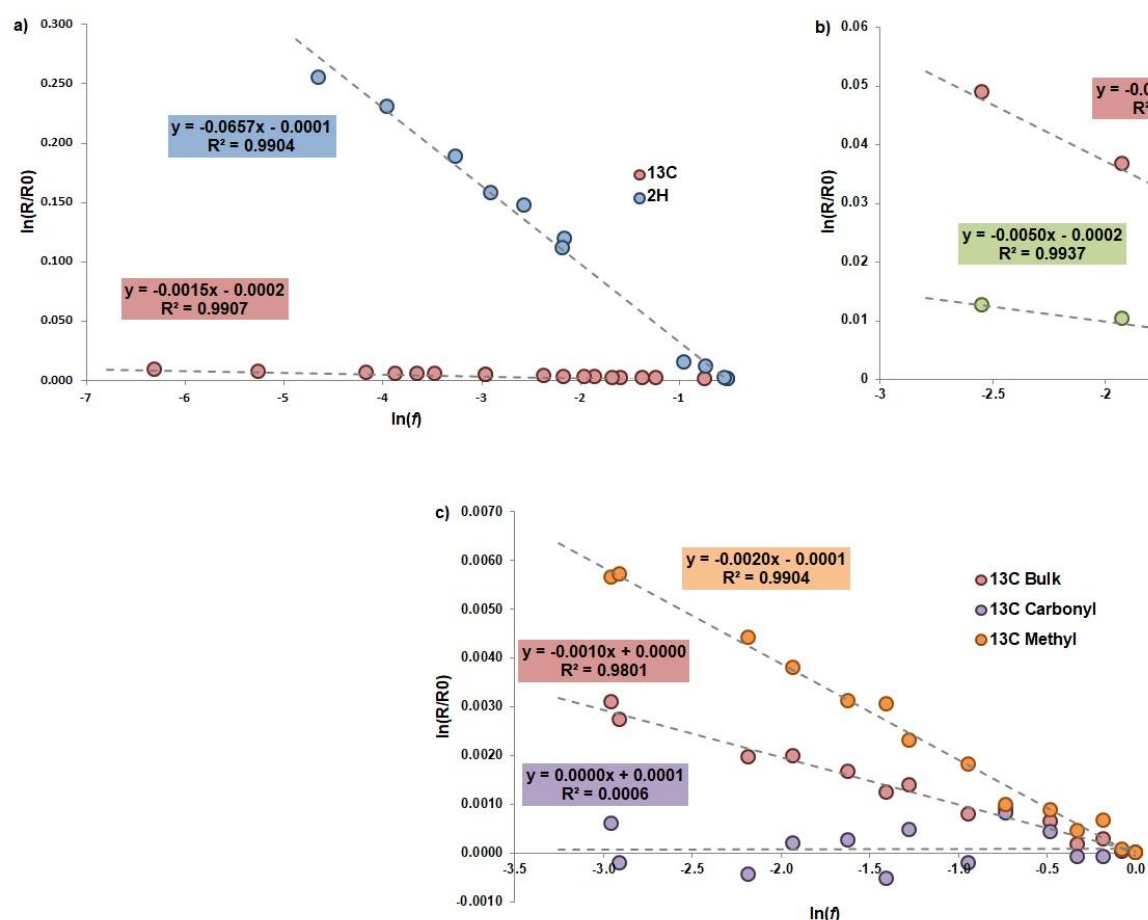

Figure 2: Rayleigh-plots drawn from experimental data of (a) Gray et al. 2002, (b)

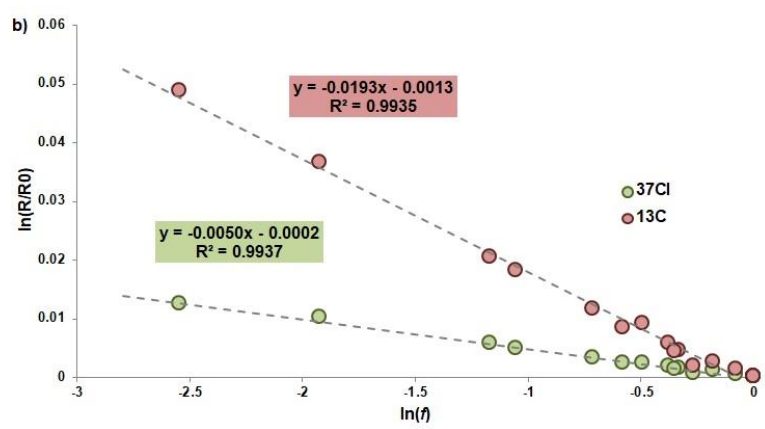

497

498

499

500

501 
503

504

505

506

507
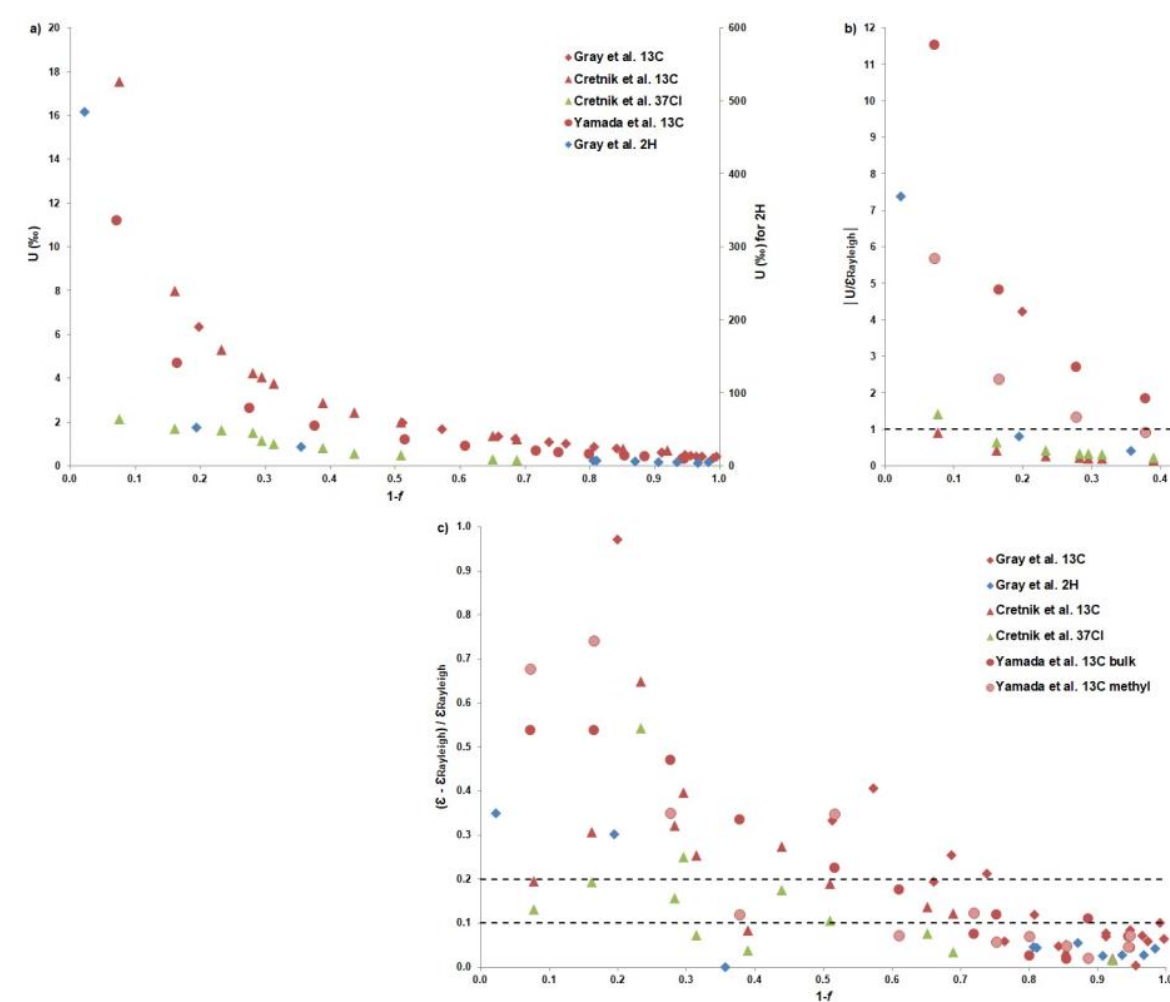

Figure 3: Projection of the transformation yield ( $x$-axis expressed as $1-f$ ) and on the y-axis (a) $U$, (b) $\left|U / \varepsilon_{\text {Rayleigh }}\right|$ and (c) $\left(\varepsilon-\varepsilon_{\text {Rayleigh }}\right) / \varepsilon_{\text {Rayleigh. }}$. Dotted lines correspond to (b) the significance threshold $\left(\left|U / \varepsilon_{\text {Rayleigh }}\right|=1\right)$ and (c) the gap between values obtained using the two-points calculation and the true value (fixed at 10 and 20\%).

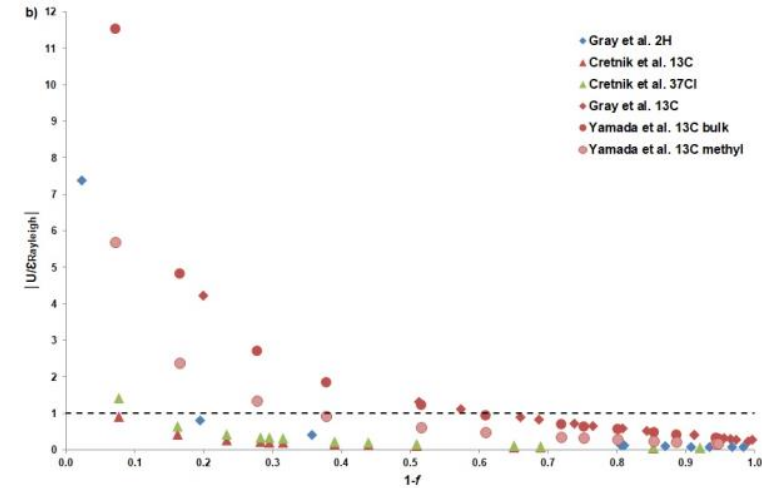

